\title{
CT and MRI of diffuse lobar involvement pattern in liver pathology
}

\author{
Muşturay Karçaaltıncaba, Claude B. Sirlin
}

\begin{abstract}
Focal, segmental, and diffuse liver pathologies have been described in the literature. This article describes a pattern in which liver pathology is confined to a lobe. This lobar pattern has not been described previously to our knowledge. Herein, we illustrate computed tomography (CT) and magnetic resonance imaging (MRI) findings of diffuse lobar involvement patterns in various liver conditions. Diffuse lobar involvement can be observed in benign (steatosis, hepatic iron overload, cholestasis, perfusion alterations, infarction, alveolar hydatid cysts, trauma, and hemangiomas) and primary malignant (hepatocellular carcinoma) pathologies. Diffuse lobar involvement in metastatic disease appears to be rare. Due in part to their potentially unusual appearances, the diagnosis of lobar pathologies using imaging can be challenging, and entities with lobar patterns can cause diagnostic confusion. Liver MRI can be used as a problem-solving tool for diffuse lobar pathologies detected on ultrasonography and CT. Inand out-of-phase MRI can help in the assessment of lobar fat accumulation.
\end{abstract}

Key words: • liver $\bullet$ computed tomography $\bullet$ magnetic resonance imaging
From the Department of Radiology (M.K. $ه$ musturayk@ yahoo.com), Hacettepe University School of Medicine, Ankara, Turkey; and the Department of Radiology (C.B.S.), University of California at San Diego, San Diego, California, USA.

Received 25 October 2010; accepted 26 October 2010.

Published online 5 November 2010

DOI 10.4261/1305-3825.DIR.4033-10.0
$\mathbf{F}$ ocal, segmental, and diffuse liver pathologies have been described in the literature. We have observed lobar involvement in liver pathologies in our clinics, which is a pattern that has not been previously described in the literature. Due to their rarity and potentially unusual appearance, the imaging diagnosis of lobar pathologies can be challenging, and entities with lobar patterns can cause diagnostic confusion. This pictorial essay illustrates computed tomography (CT) and magnetic resonance imaging (MRI) findings of diffuse lobar involvement patterns in several liver conditions. Conditions with periportal distributions (e.g., neurofibromatosis, perivascular fatty infiltration, periportal edema, and cavernomatous transformation) within a lobe have been previously described (1) and are not illustrated here.

\section{Hemangioma}

Hemangiomas usually appear as distinct masses with rounded contours. Hemangiomas can be giant and can fill most of a hepatic lobe (Fig. 1), and the recognition of this type of lesion as a distinct mass is usually straightforward because they do not conform to the lobe $(2,3)$. Rarely, hemangiomas may conform to a hepatic lobe and present with a diffuse lobar pattern (Fig. 2). While the morphologies of diffuse lobar hemangiomas may cause confusion, characteristic imaging features facilitate a correct diagnosis. These include centripedal nodular contrast enhancement that approximates that of the blood pool in attenuation or intensity as well as marked hyperintesity on T2-weighted MR images. MRI is helpful for diagnosing hemangiomas in patients with equivocal findings on CT or ultrasonography (US).

\section{Hepatocellular carcinoma}

Hepatocellular carcinomas usually manifest as focal masses or nonmasslike infiltrative processes $(4,5)$. Diffuse involvement of the left or right lobes by hepatocellular carcinoma is rare and may be difficult to diagnose by CT or ultrasonography. MRI may elucidate lobar involvement with hepatocellular carcinoma (Figs. 3 and 4) by depicting arterialphase hyper-enhancement, venous-phase wash-out or heterogeneity, abnormal signal intensity on T1-weighted images, and mild-moderate hyperintensity on T2-weighted and diffusion-weighted images. In- and out-of-phase images may be helpful in showing either relative fat sparing or preferential fat accumulation in the affected lobe.

\section{Infection}

Hydatid cyst disease can be caused by echinoccus granulosus and echinococcus alveolaris. Infection of the liver with alveolar hydatid cysts can mimic a malignant mass and lead to venous invasion and biliary dilatation (6). CT (Fig. 5) or MR images can reveal a heterogeneous cystic mass 

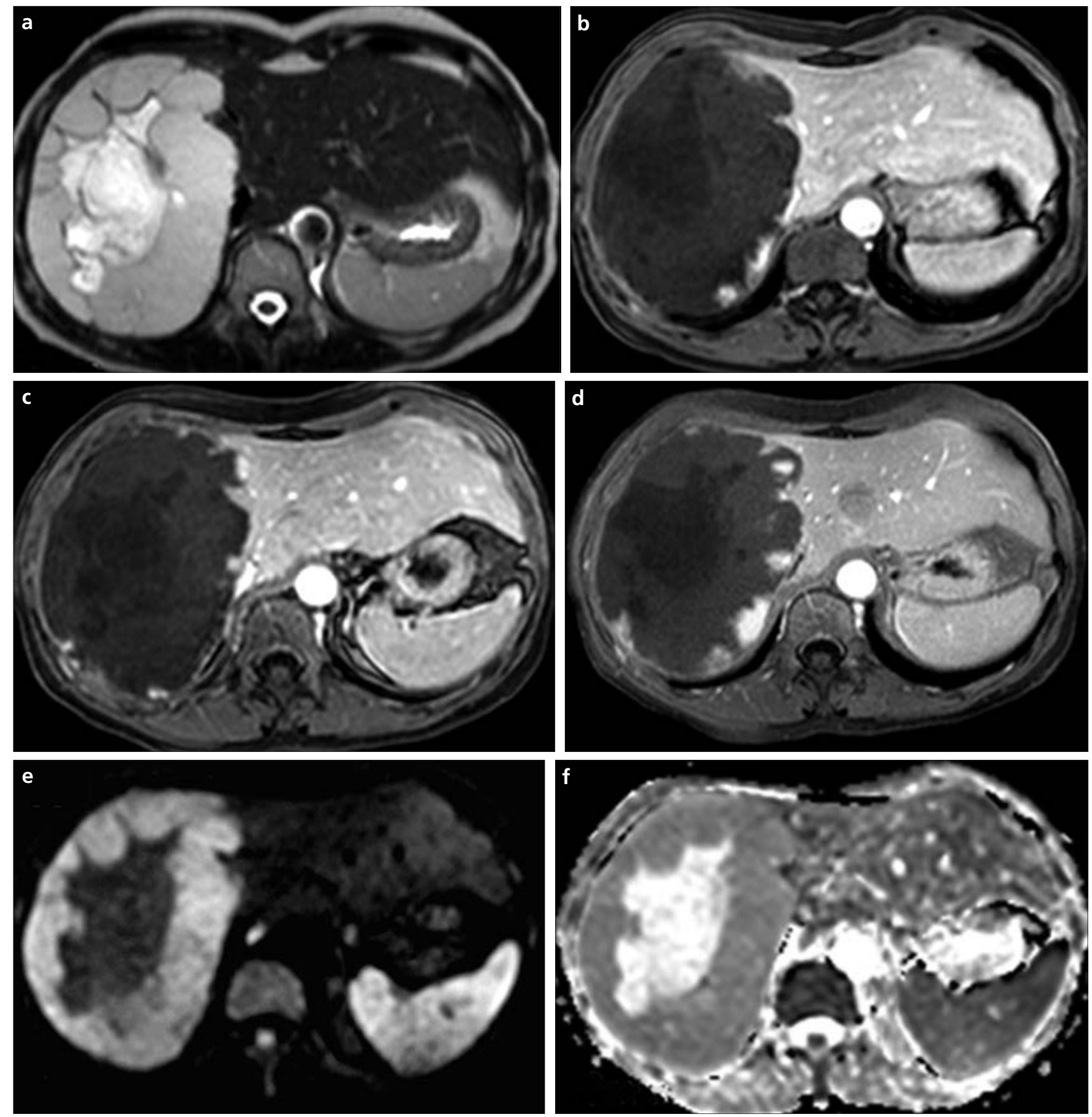

Figure 1. a-f. A 50 year-old woman with a liver mass that was diagnosed by ultrasonography. Axial T2-weighted image depicts hyperintense lesion localized in the right lobe (a). Post-contrast images (b-d) demonstrate peripheral nodular enhancement and centripetal contrast filling, which confirms the diagnosis of hemangioma. Note the central presence of the scar-like hyperintense cystic component. The diffusion-weighted image (e) and apparent diffusion coefficient map (f) show high diffusivity in the cystic component.

that is limited to the right or left lobes. The diagnosis of a hydatid cyst can be suggested by a lack of enhancement of a solid-cystic mass and the presence of calcification. Biopsy may be required for diagnosis.

\section{Infarction}

Infarction of the liver is usually due to trauma (Fig. 6) and usually occurs as a result of the laceration of blood vessels that supply the right or left lobes; however, this condition has also been shown to occur as a result of other causes (toxic or viral hepatitis, nontraumatic occlusion of both the hepatic artery and portal vein branch that supplies the affected lobe, idiopathic sources, etc.) (Fig. 7) (7-9). 

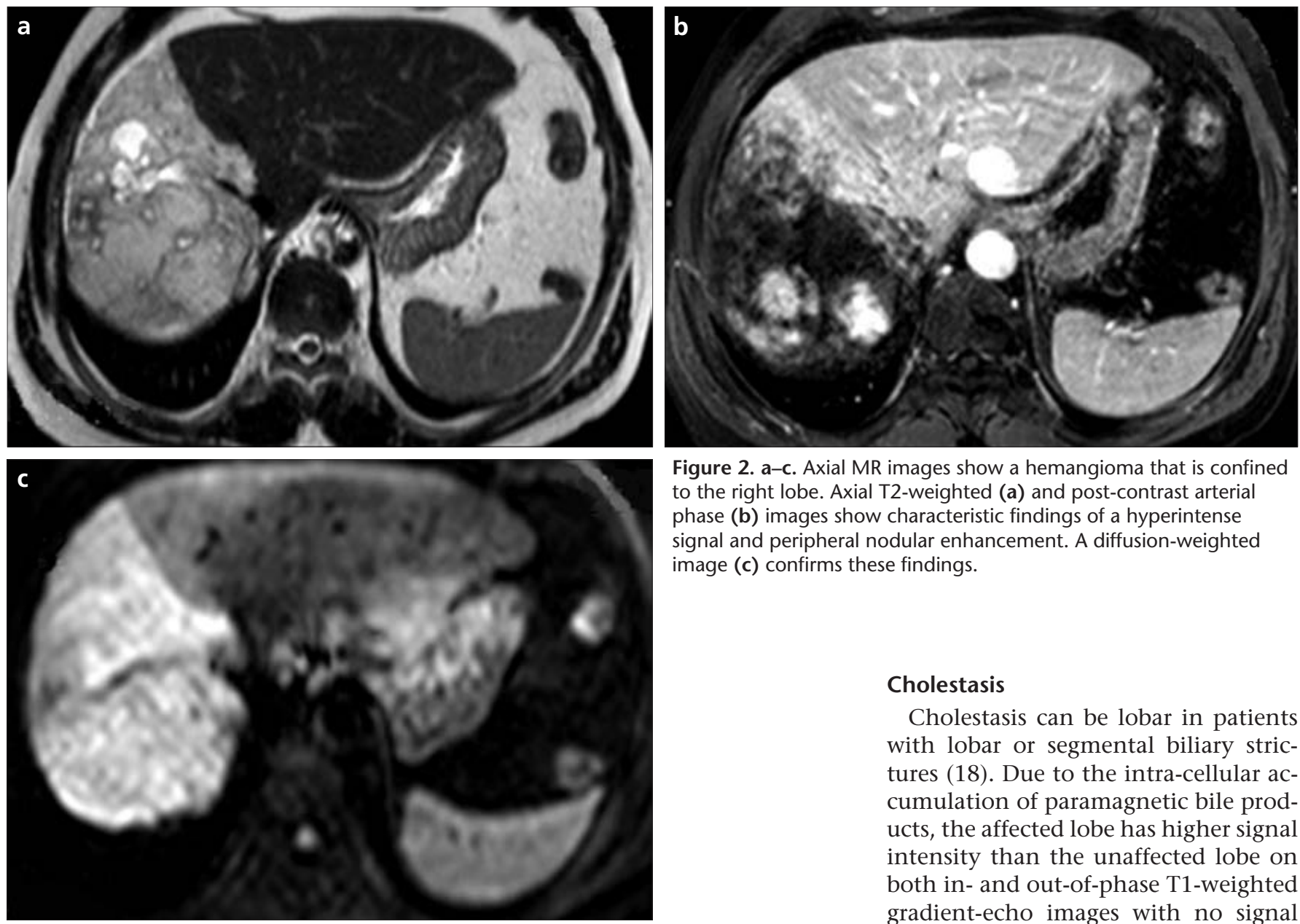

Figure 2. a-c. Axial MR images show a hemangioma that is confined to the right lobe. Axial T2-weighted (a) and post-contrast arterial phase (b) images show characteristic findings of a hyperintense signal and peripheral nodular enhancement. A diffusion-weighted image (c) confirms these findings.

\section{Perfusion changes}

The left or right portal veins can be occluded, leading to a transient hepatic attenuation difference that is only detectable on arterial phase images (Fig. $8)(10,11)$. In hypereosinophilic syndrome, a lobar involvement pattern is usually most likely caused by periportal eosinophil infiltration and the resultant portal vein stenosis (12).

\section{Fat accumulation}

Fat accumulation in the liver is usually diffuse but can also be focal or heterogeneous (13-15). Lobar fat accumulation is rare but can occur in patients with right or left portal vein occlusion. Lobar portal vein occlusion can change the fat content of a lobe because the portal vein brings nutrients to the liver from the gastrointestinal tract. Lobar fat accumulation can also be idiopathic. The diagnosis may be difficult on US or CT but is usually straightforward on MRI; in- and out-of-phase images can be diagnostic for lobar fat accumulation by demonstrating signal drop on out-of-phase images preferentially or exclusively in the affected lobe (Fig. 9).

The diagnosis of lobar fat accumulation in patients with underlying iron overload is challenging even with MRI; however, careful analysis of inand out-of-phase T1-weighted gradient-echo images may direct a correct diagnosis. On out-of-phase images, lobes that contain both fat and iron are likely to appear more hypointense in comparison to lobes that only contain iron (Fig. 10). On in-phase images, the signal intensity difference between lobes is likely to be smaller. Advanced MR imaging techniques that are designed to simultaneously assess fat and iron accumulation are now being developed $(16,17)$. These techniques are likely to facilitate the diagnosis of lobar fat accumulation that is superimposed over iron overload; however, these techniques are not yet commercially available.

\section{Cholestasis}

Cholestasis can be lobar in patients with lobar or segmental biliary strictures (18). Due to the intra-cellular accumulation of paramagnetic bile products, the affected lobe has higher signal intensity than the unaffected lobe on both in- and out-of-phase T1-weighted gradient-echo images with no signal drop on out-of-phase images (Fig. 11). Thus, cholestasis can be easily differentiated from lobar fat accumulation. If a hepatobiliary agent (e.g., gadoxetate) is administered, the affected lobe exhibits reduced signal intensity in the hepatocellular phase due to an impaired uptake of the agent (19). Lobar cholestasis is difficult to diagnose using CT or US, although the presence of dilated intrahepatic ducts in a lobar distribution may suggest the diagnosis.

As a conclusion, liver pathologies may manifest with lobar distributions. Such manifestations are uncommon and may cause diagnostic confusion. The differential diagnoses of these pathologies include benign and primary malignant etiologies. Diffuse lobar involvement via metastatic disease appears to be rare, and US findings are usually nonspecific. While CT findings may be diagnostic, they are often not definitive. In such cases, MR imaging may be helpful in achieving the correct diagnosis. Awareness of specific lobar patterns in combination with a careful review of MRI features can usually lead to a correct diagnosis. 

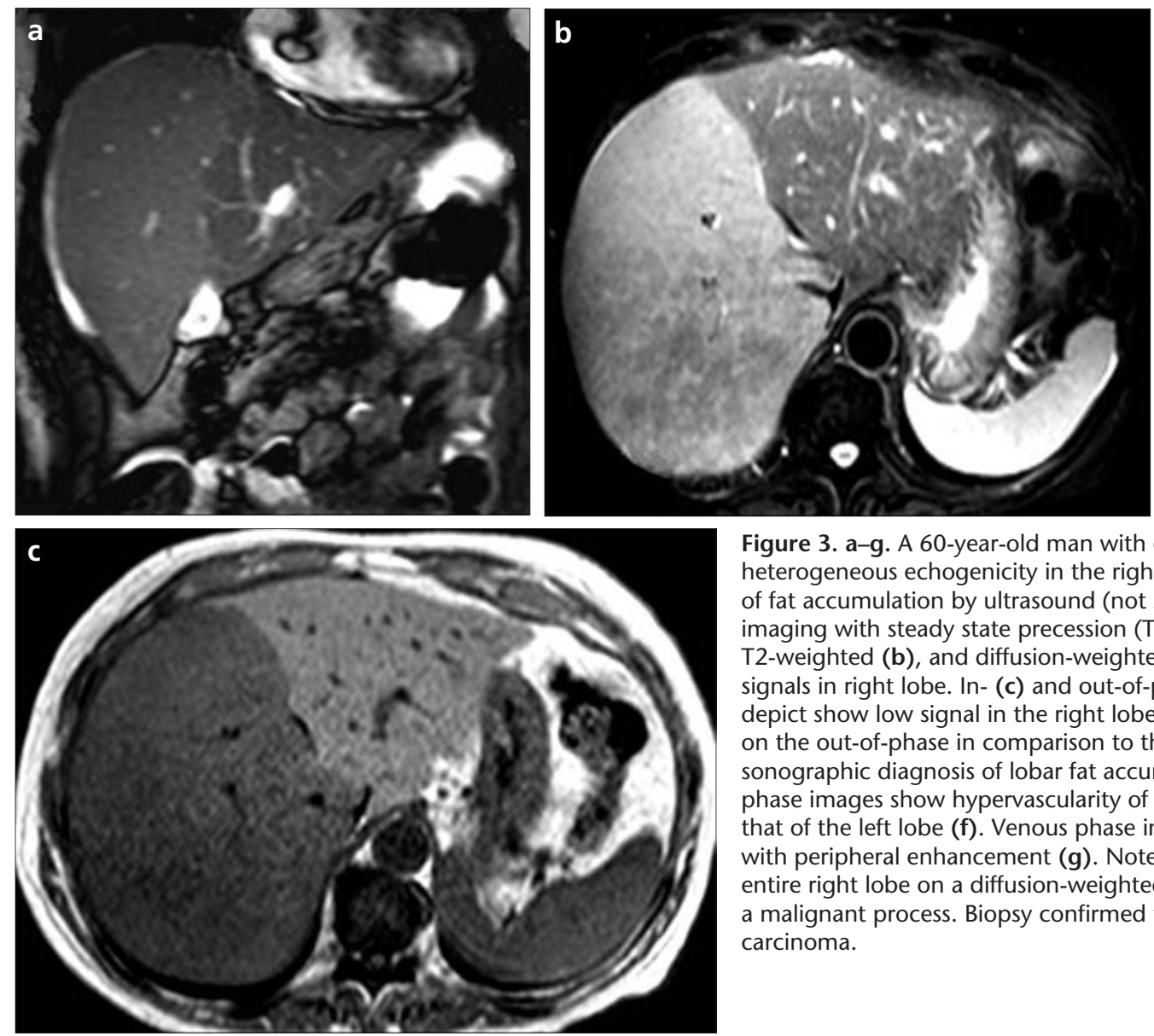

Figure 3. a-g. A 60-year-old man with elevated liver enzymes with heterogeneous echogenicity in the right lobe of the liver with suspicion of fat accumulation by ultrasound (not shown). Coronal true fast imaging with steady state precession (TRU-FISP) (a), fat saturated axial T2-weighted (b), and diffusion-weighted (e) images show diffuse high signals in right lobe. In- (c) and out-of-phase (d) gradient echo images depict show low signal in the right lobe. The absence of signal loss on the out-of-phase in comparison to the in-phase image refutes the sonographic diagnosis of lobar fat accumulation. Post-contrast arterial phase images show hypervascularity of the right lobe in comparison to that of the left lobe (f). Venous phase images show heterogeneous signal with peripheral enhancement $(\mathbf{g})$. Note the high signal intensity of the entire right lobe on a diffusion-weighted image (e). MRI findings suggest a malignant process. Biopsy confirmed the diagnosis of hepatocellular carcinoma.
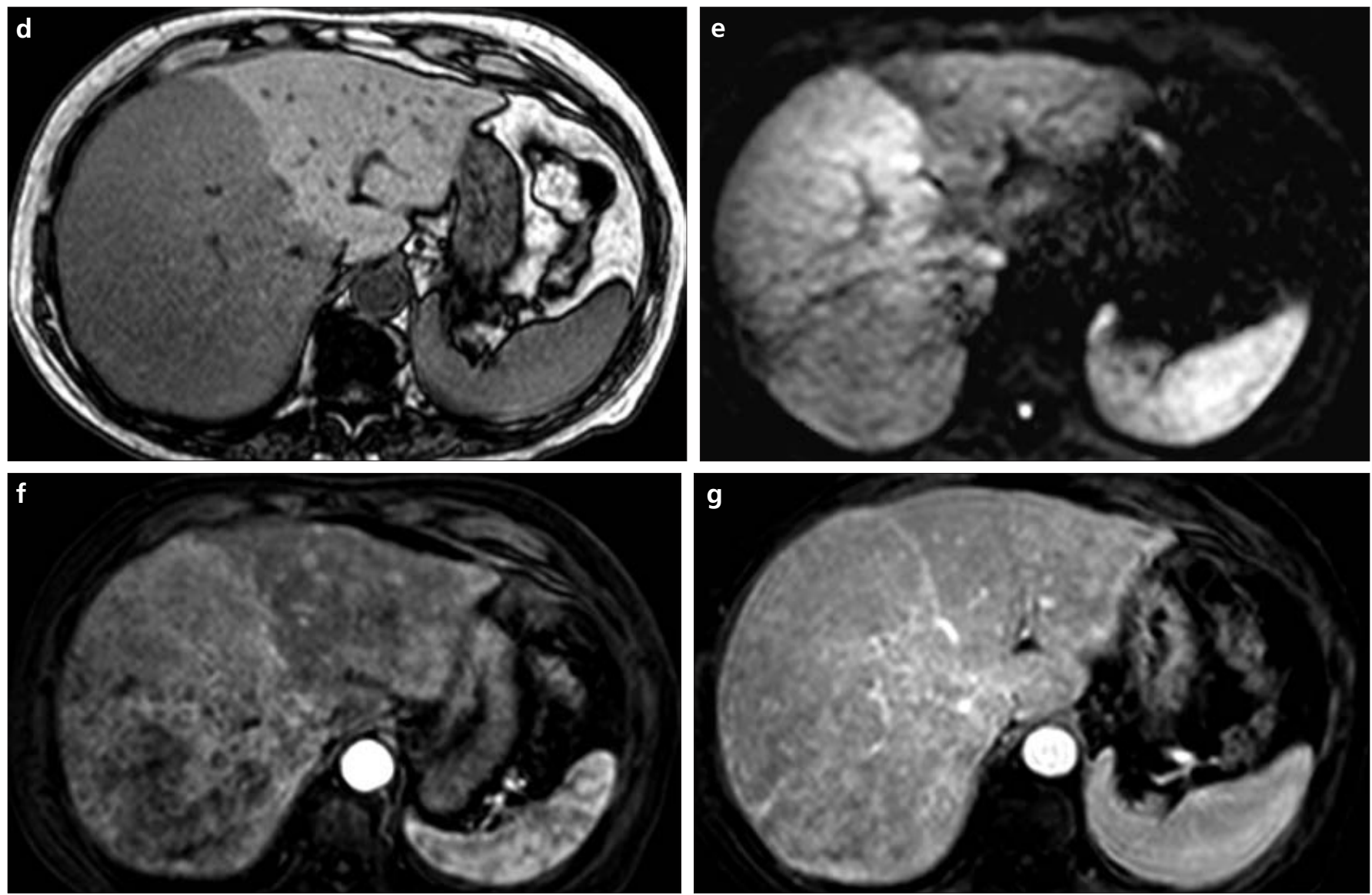

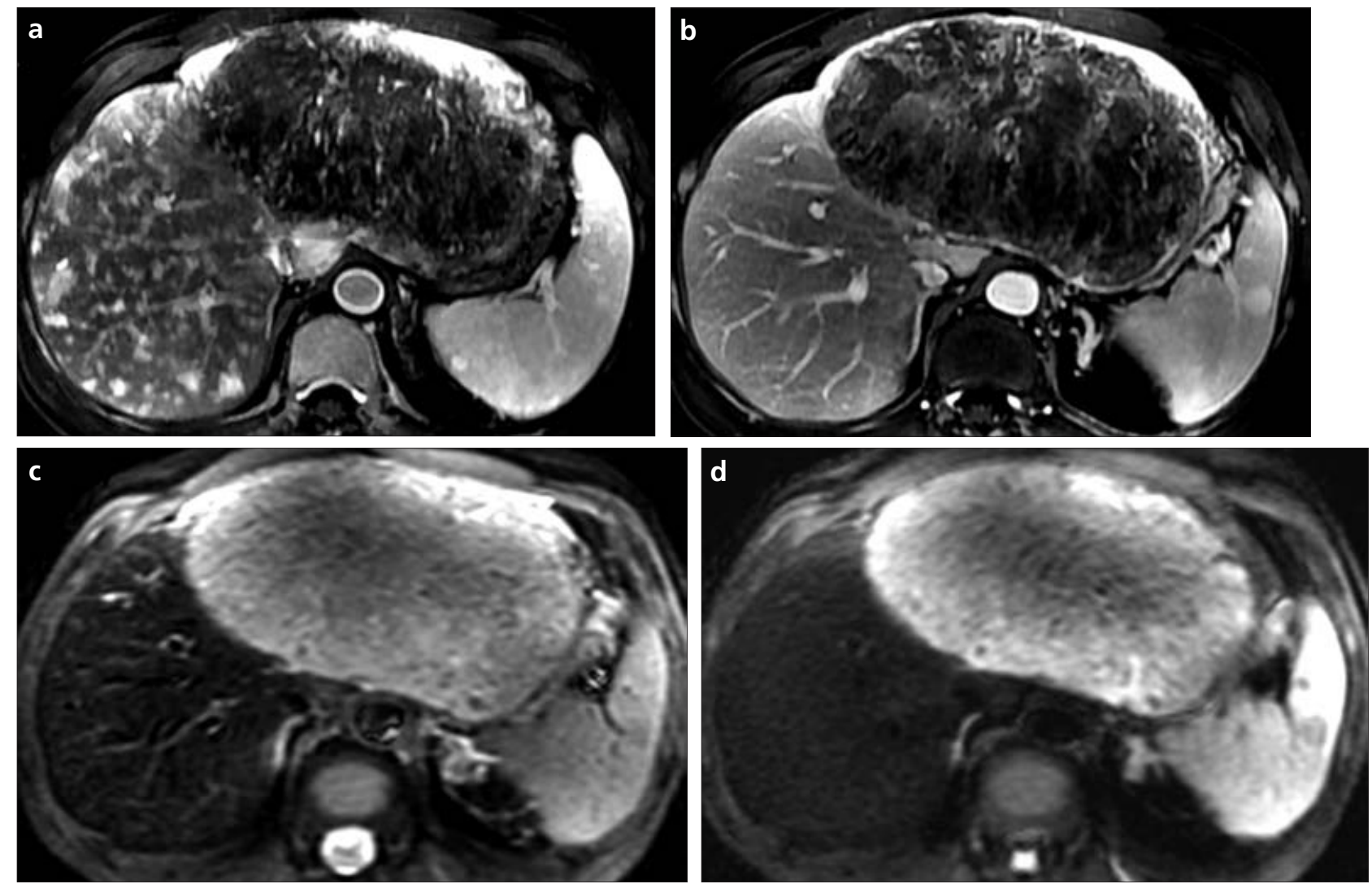

Figure 4. a-d. A 54 year-old man with chronic hepatitis B viral infection presented with a palpable abdominal mass. Hepatic arterial phase (a) and portal venous phase (b) MR images at $1.5 \mathrm{~T}$ reveal a heterogeneous mass that occupied the entire left lobe. Note the abnormal vessels within the mass and the surrounding tumor capsule, which can be best seen on the portal venous phase image. Innumerable arterial-phase hyper-enhancing, ill-defined, and vaguely nodular perfusional alterations are present in the right lobe; these pseudolesions are probably due to arterioportal shunts. These pseudolesions fade to iso-intensity on the portal venous phase. Diffusion-weighted images with b-values of 0 (c) and 500 (d) s/mm² show low diffusivity within the mass. Note that the right lobe of the liver appears to be normal on the diffusion-weighted images; the pseudolesions are not visible.
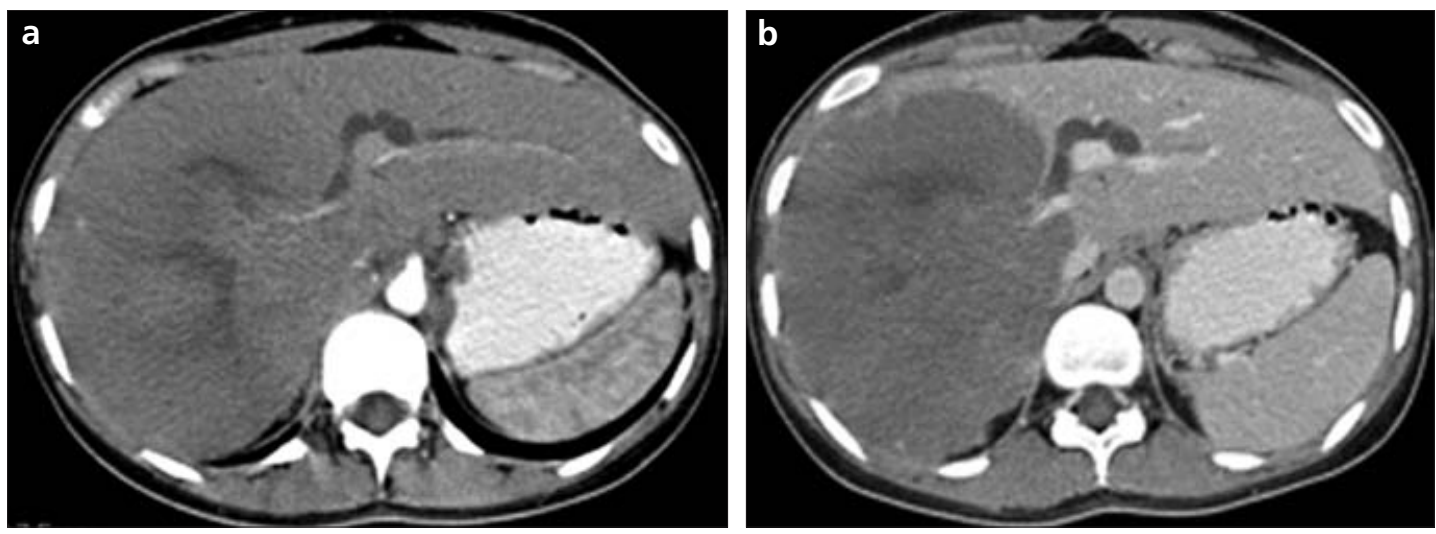

Figure 5. a-d. A 21-yearold woman with a right liver lobe mass detected on ultrasonography. Axial CT images (a-d) show a hypodense mass with calcifications that are limited to the right lobe and causing biliary dilatation of the left lobe. Biopsy confirmed the diagnosis of alveolar echinoccosis. The patient underwent a right hepatectomy. Note the presence of calcifications
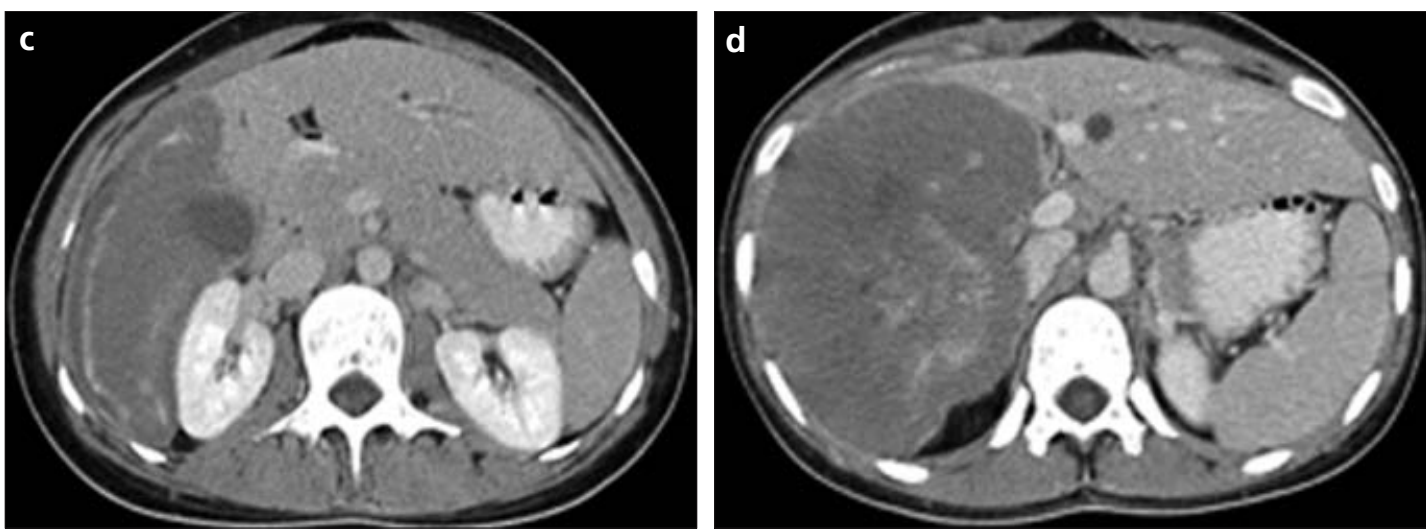
(c, d) and a lack of enhancement. 


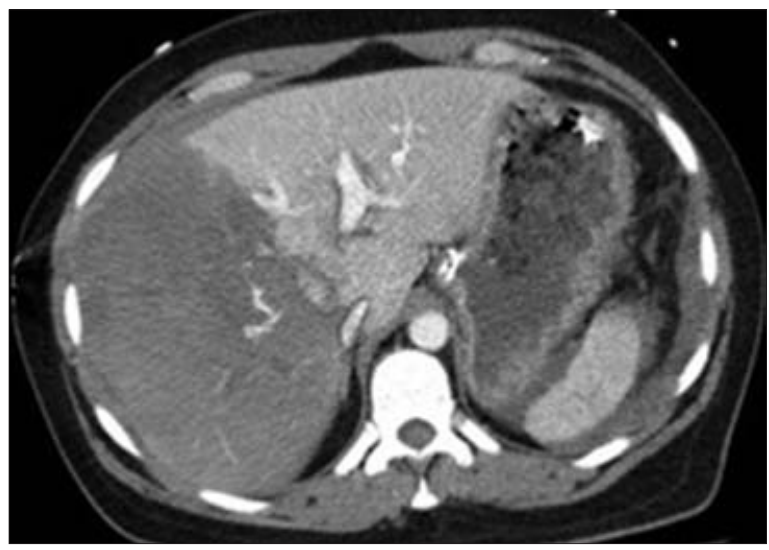

Figure 6. A 50-year-old man who had sustained

trauma. Axial CT image shows a lack of perfusion

to the entire right lobe due to traumatic infarction.
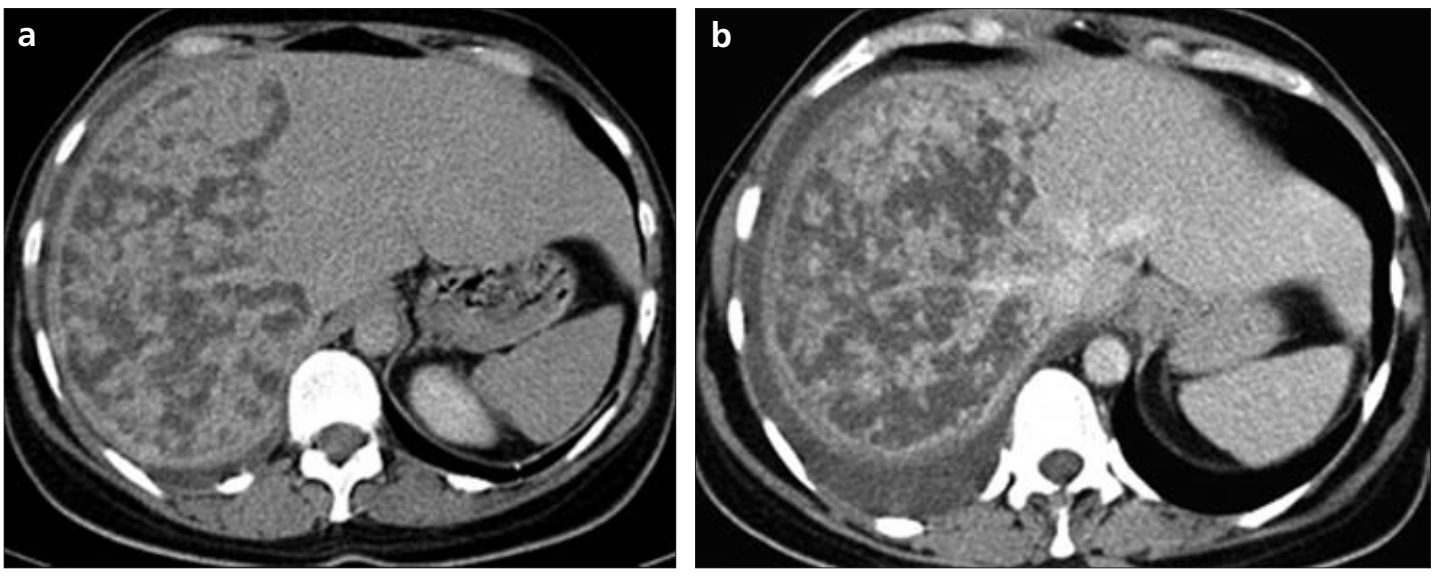

Figure 7. a-f. A 41-yearold woman presented with right upper quadrant pain after cesarean section. Axial CT images $(a, b)$ show capsular enhancement and diffuse patchy hypodense areas exclusively in the right lobe. Axial T2-weighted (c) and diffusionweighted (d) images depict hyperintensity and restricted diffusion. Axial in- (e) and out-of-phase (f) T1-weighted images exclude the diagnosis
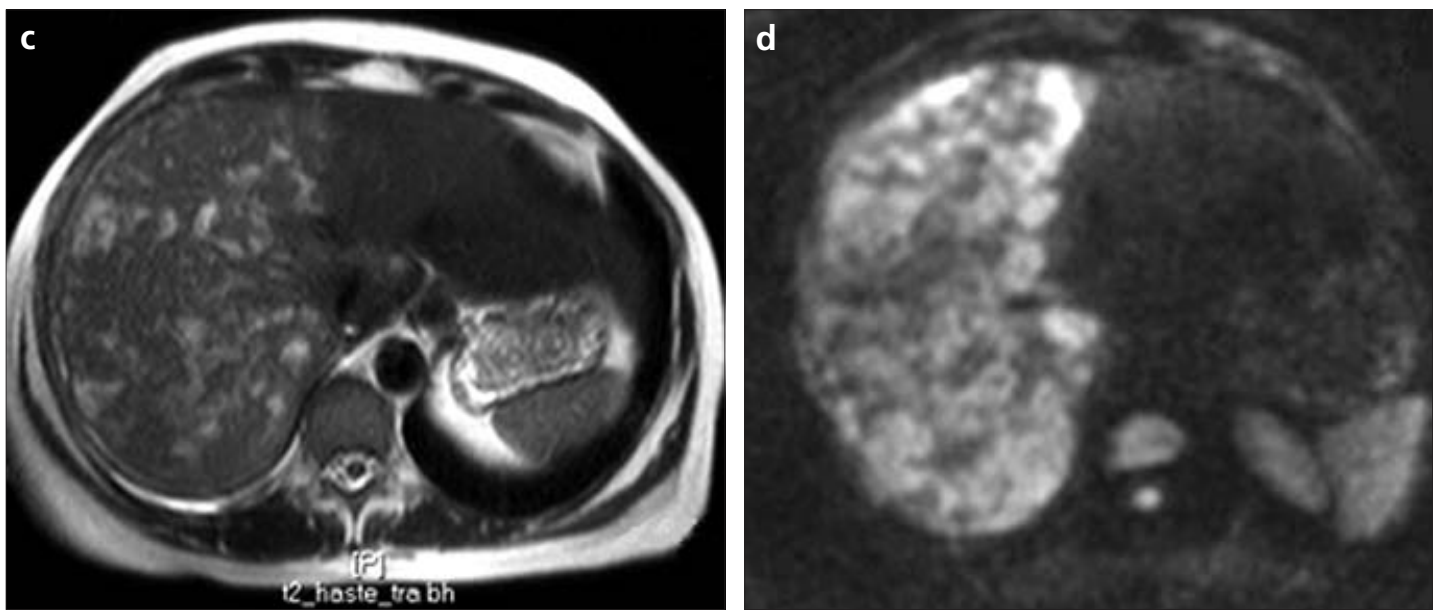
of heterogeneous fat accumulation. Right liver lobe infarction is suggested based on imaging findings and the spontaneous resolution of pain within a week. The infarction was attributed to non-traumatic thrombosis of vessels supplying the right lobe.
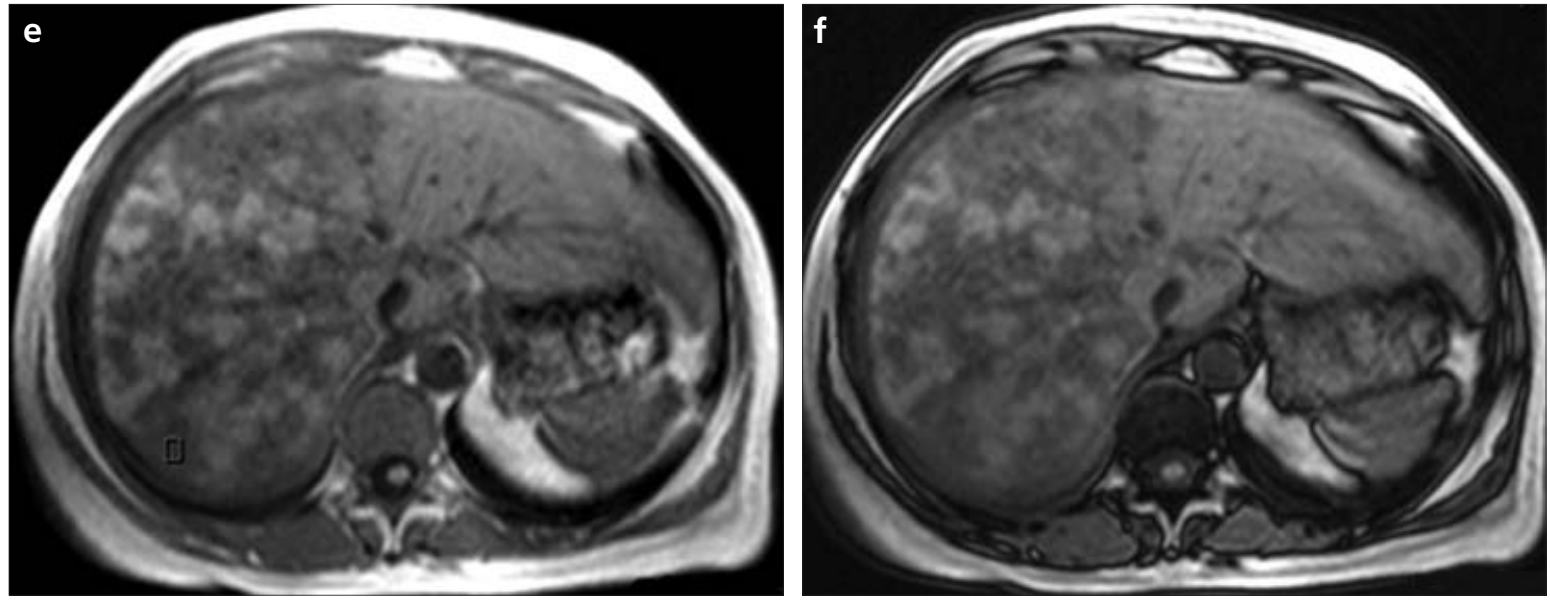

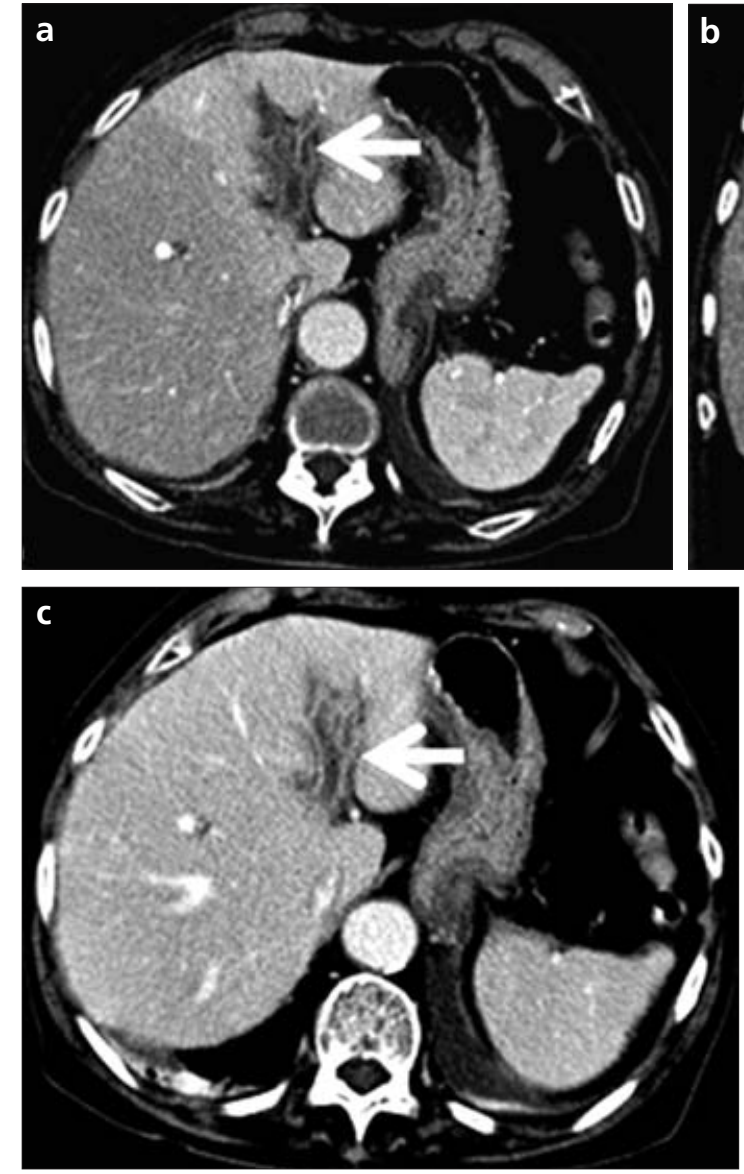

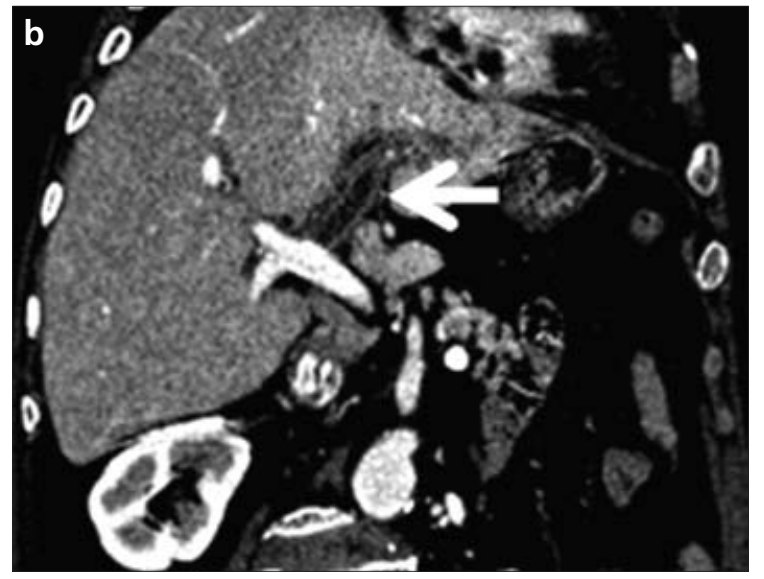

Figure 8. a-c. Axial (a) and coronal (b) late arterial phase CT images depict a diffuse transient hepatic attenuation difference in the left lobe due to left portal vein occlusion (arrows). The density difference disappears on the venous phase image $($ arrow, c). Note the relatively small size of the left lobe, presumably due to atrophy from long-standing lack of portal venous supply.
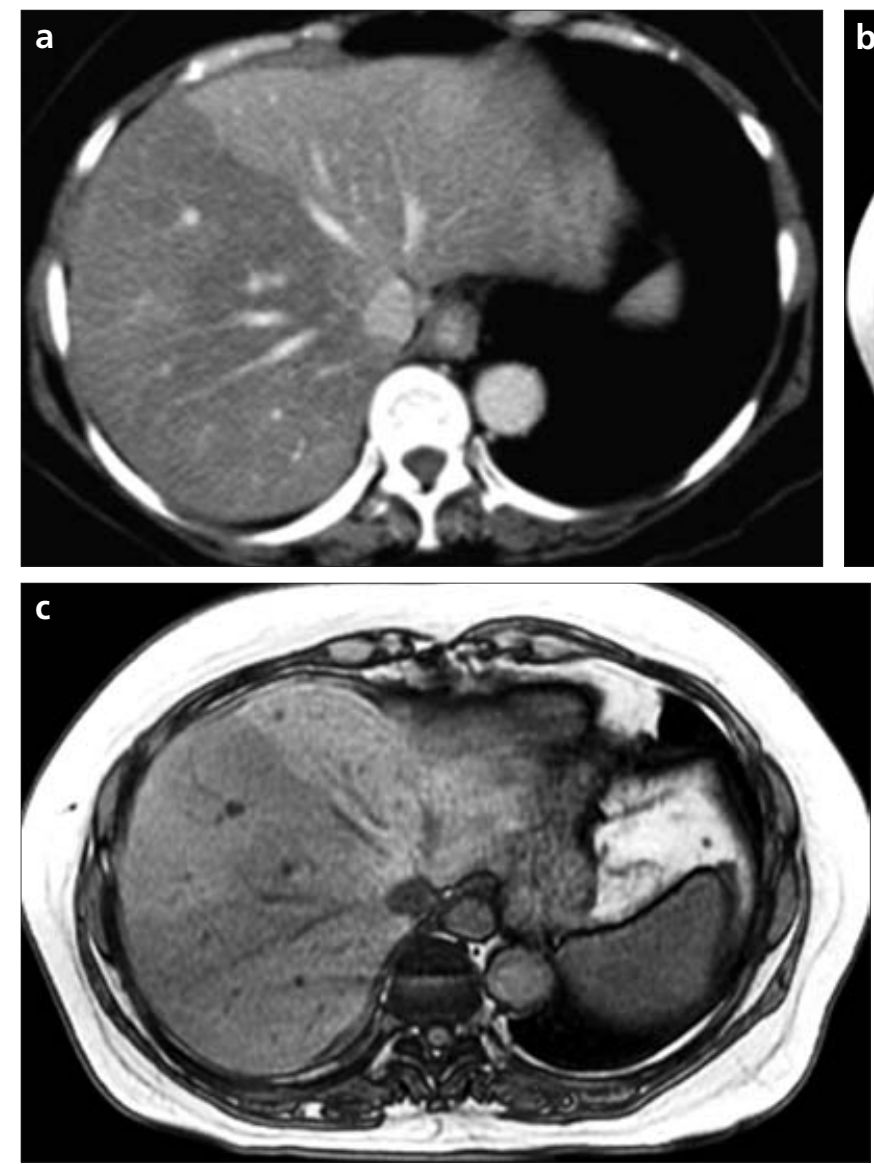

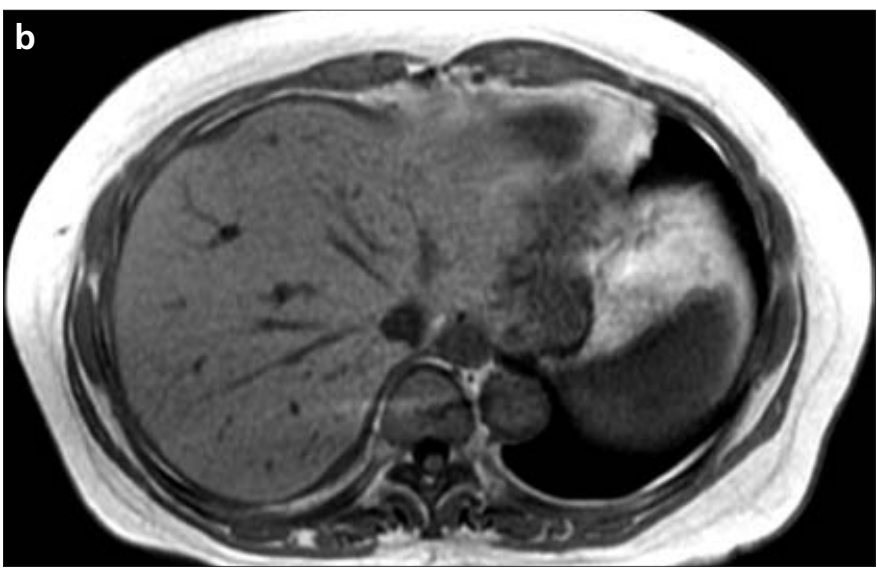

Figure 9. a-c. A 49-year-old woman who was operated on due to the presence of a periampullary tumor. The axial CT image (a) shows hypodensity of the right lobe in comparison to left lobe, which is consistent with fat accumulation. The differential diagnosis from the findings on this CT image includes a transient hepatic attenuation difference; however, in this case, the attenuation difference persisted on other phases. Axial in- and out-of-phase (b, c) T1-weighted MR images show signal drop in left lobe of liver on out-of-phase images consistent with steatosis. 

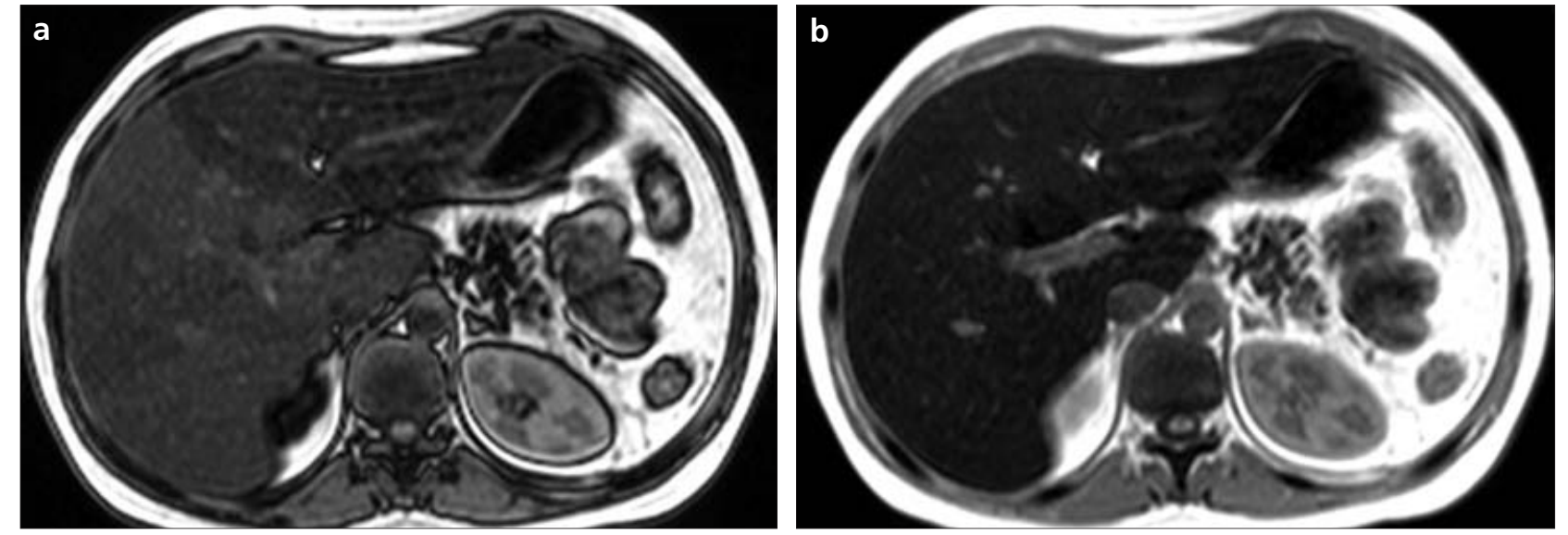

Figure 10. a, b. A 19-year-old man with hemosiderosis and left lobar fat accumulation. Axial out-of-phase MR image (a) shows hypointensity of the left lobe (due to iron and fat deposition) compared to the right lobe (due to only iron deposition). An in-phase T1-weighted MR image (b) shows no difference between the right and left lobes. Note that the signal from the liver on the in-phase is lower compared to a normal liver due to iron overload.
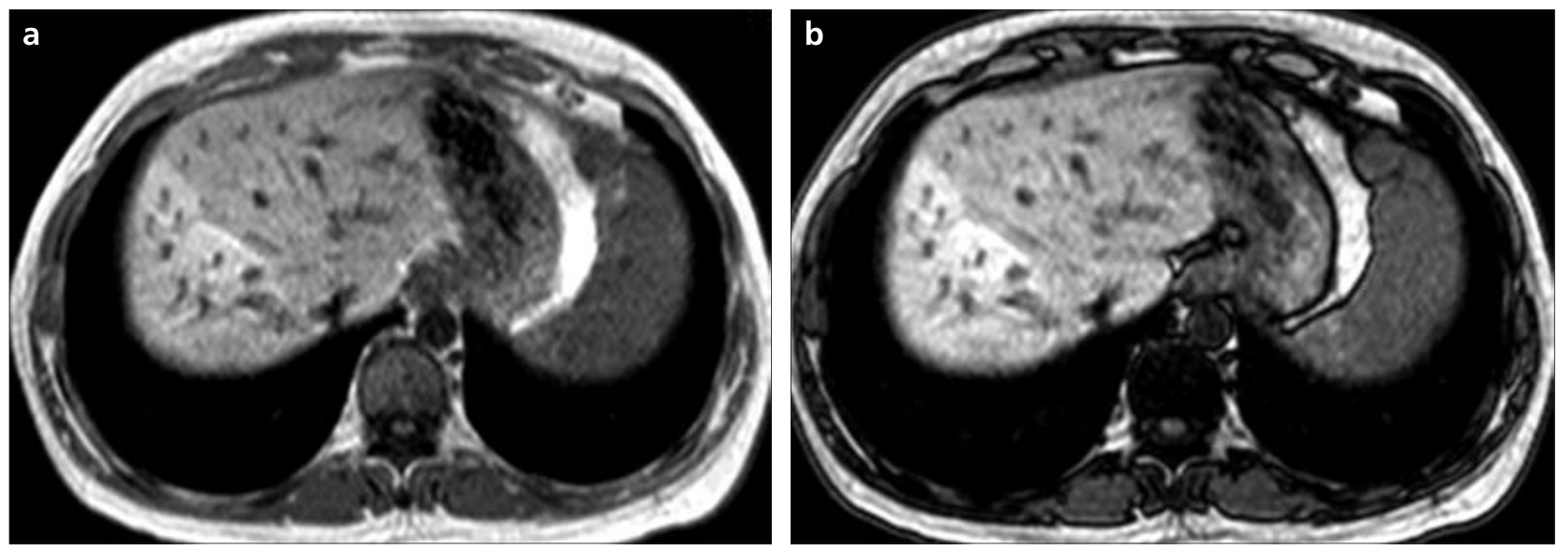

Figure 11. a, b. A 34-year-old woman with sclerosing cholangitis. The right lobe appears more hyperintense compared to the left lobe on the in phase T1-weighted MR image (a) due to cholestasis. No signal drop is seen in the out-of-phase MR image (b).

\section{Conflict of interest disclosure}

The authors declared no conflicts of interest.

\section{References}

1. Karcaaltincaba M, Haliloglu M, Akpinar E, et al. Multidetector CT and MRI findings in periportal space pathologies. Eur J Radiol 2007; 61:3-10.

2. Coumbaras M, Wendum D, MonnierCholley L, Dahan H, Tubiana JM, Arrivé L. CT and MR imaging features of pathologically proven atypical giant hemangiomas of the liver. AJR Am J Roentgenol 2002; 179:1457-1463.

3. Danet IM, Semelka RC, Braga L, Armao D, Woosley JT. Giant hemangioma of the liver: MR imaging characteristics in 24 patients. Magn Reson Imaging 2003; 21:95101.

4. Hanna RF, Kased N, Kwan SW, et al. Double-contrast MRI for accurate staging of hepatocellular carcinoma in patients with cirrhosis. AJR Am J Roentgenol 2008; 190:47-57.
5. Willatt JM, Hussain HK, Adusumilli $S$ Marrero JA. MR Imaging of hepatocellular carcinoma in the cirrhotic liver: challenges and controversies. Radiology 2008; 247:311-330.

6. Rozanes I, Acunaş B, Celik L, Minareci O, Gökmen E. CT in lobar atrophy of the liver caused by alveolar echinococcosis. J Comput Assist Tomogr 1992; 16:216-218.

7. Cakir B, Teksam M, Tarhan NC, et al. Unusual MDCT and sonography findings in fulminant hepatic failure resulting from hepatitis A infection. AJR Am J Roentgenol 2005; 185:1033-1035.

8. Giovine S, Pinto A, Crispano S, Lassandro F, Romano L. Retrospective study of 23 cases of hepatic infarction: CT findings and pathological correlations. Radiol Med 2006; 111:11-21.

9. Taourel P, Vernhet H, Suau A, Granier C, Lopez FM, Aufort S. Vascular emergencies in liver trauma. Eur J Radiol 2007; 64:7382.
10. Lee KH, Han JK, Jeong JY et al. Hepatic attenuation differences associated with obstruction of the portal or hepatic veins in patients with hepatic abscess. AJR Am J Roentgenol 2005; 185:1015-1023.

11. Colagrande S, Carmignani L, Pagliari A, Capaccioli L, Villari N. Transient hepatic attenuation differences (THAD) not connected to focal lesions. Radiol Med 2002; 104:25-43.

12. Lim JH, Lee WJ, Lee DH, Nam KJ. Hypereosinophilic syndrome: CT findings in patients with hepatic lobar or segmental involvement. Korean J Radiol 2000; 1:98103.

13. Hamer OW, Aguirre DA, Casola G, Lavine JE, Woenckhaus M, Sirlin CB. Fatty liver: imaging patterns and pitfalls. Radiographics 2006; 26:1637-1653.

14. Karcaaltincaba M, Akhan O. Imaging of hepatic steatosis and fatty sparing. Eur J Radiol 2007; 61:33-43. 
15. Merkle EM, Nelson RC. Dual gradientecho in-phase and opposed-phase hepatic MR imaging: a useful tool for evaluating more than fatty infiltration or fatty sparing. Radiographics 2006; 26:14091418.

16. Yokoo T, Bydder M, Hamilton G, et al. Nonalcoholic fatty liver disease: diagnostic and fat-grading accuracy of low-flip-angle multiecho gradient-recalled-echo MR imaging at 1.5 T. Radiology 2009; 251:67-76.
17. Yu H, McKenzie CA, Shimakawa A, et al. Multiecho reconstruction for simultaneous water-fat decomposition and $\mathrm{T} 2{ }^{*}$ estimation. J Magn Reson Imaging. 2007; 26:1153-1161.

18. Dave M, Elmunzer BJ, Dwamena BA Higgins PD. Primary sclerosing cholangitis: meta-analysis of diagnostic performance of MR cholangiopancreatography. Radiology 2010; 256:387-396.
19. Ringe KI, Husarik DB, Sirlin CB, Merkle EM. Gadoxetate disodium-enhanced MRI of the liver: part 1 , protocol optimization and lesion appearance in the noncirrhotic liver. AJR Am J Roentgenol 2010; 195:1328. 